\title{
Stellar populations in Luminous Red Galaxies: cosmic chronometers?
}

\author{
A. L. Ratsimbazafy ${ }^{1,4}$, C. M. Cress ${ }^{1,4}$, S. L. Blyth ${ }^{2}$, S. M. Crawford ${ }^{3}$, \\ E. A. Olivier ${ }^{1},{ }^{3}$ and K. J. van der Heyden ${ }^{2}$ \\ ${ }^{1}$ Physics Dept., Univ. of the Western Cape, Bag X17, Cape Town 7535, South Africa \\ email: raljha.a@gmail.com \\ ${ }^{2}$ Astronomy Dept., Univ. of Cape Town, Bag X3, Cape Town 7701, SA \\ ${ }^{3}$ SA Astronomical Observatory, P.O.Box 9, Observatory 7935, Cape Town, SA \\ ${ }^{4}$ Centre for High Performance Computing, 15 Lower Hope St, Rosebank, Cape Town, SA
}

\begin{abstract}
The expansion rate of the Universe can potentially be measured using age-dating of Luminous Red Galaxies (LRGs). Here, we explore the validity of the assumptions implicit in this method using LRGs identified in the Millenium Simulation (MS). We use stellar population modelling and spectral synthesis to estimate the errors on ages that can be expected and discuss optimization of such an experiment. We find that $\mathrm{H}(\mathrm{z})$ using simulated galaxies from MS can be recovered with high accuracy. Using single stellar populations (SSPs) to age-date LRGs is not sufficient but if the MS star formation histories of galaxies are used, accurate ages are obtainable. We discuss an observing program to carry out this experiment using SALT (Southern African Large Telescope).
\end{abstract}

Keywords. galaxies - galaxies: evolution - cosmology parameters - cosmology: observations

\section{Identifying LRGs in Millenium Simulation \& Recovering H(z)}

Assuming stars in LRGs form at the same time, age-dating of two populations of LRGs at different redshifts can provide an estimate of the time difference associated with the corresponding redshift interval. This gives a direct estimate of the Hubble parameter at the average redshift of the two populations (Jimenez \& Loeb 2003). Here (and in Crawford et al. 2009), we explore the uncertainties on $\mathrm{H}(\mathrm{z})$ measured in this way by extracting LRGs from the MS. Initially, we used the SDSS LRG criteria defined by Eisenstein et al. (2001) but these apparent-magnitude cuts are defined over a wide range of redshifts and near our target redshift of $z \sim 0.5$; the MS sample of LRGs is contaminated by galaxies that have quite varied star formation histories (SFHs). We find absolute magnitude cuts in the rest frame select a more homogeneous sample of LRGs, and used $M_{V}<-23$ and $B-V>0.81$. This approach would be feasible in a real age-dating experiment given the availability of low-resolution spectra of large numbers of LRGs.

We explored the uncertainties on $\mathrm{H}(\mathrm{z})$ related to the extended $\mathrm{SFH}$ of LRGs using LRGs in different MS snapshots in the range $0<z<1$. Each pair of snapshots in MS provides an estimate of $\mathrm{H}(\mathrm{z})$ near the centre of the redshift interval. We assume the error in $\mathrm{H}(\mathrm{z})$ depends only on the errors on age at the two redshifts. We find that in the MS, as shown in Fig. 1, we can recover the $\mathrm{H}(\mathrm{z})$ implied by the cosmological parameters of the simulation using the ages of the LRGs . 


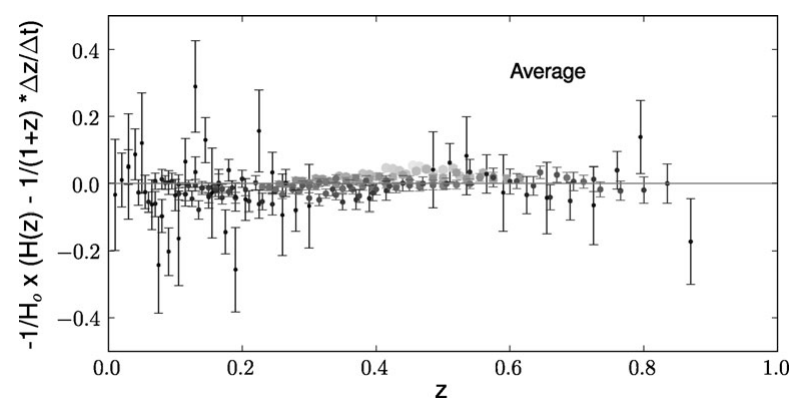

Figure 1. H(z) calculated from the average mass-weighted-age. The size of each point is related to the distance between the redshift snapshots. Larger errors are typically associated with smaller redshifts intervals. $\mathrm{H}(\mathrm{z})$ can be calculated from mass-weighted ages to a precision of less than $3 \%$.

\section{Modelling \& age-dating LRGs spectra}

Using both the SFH and metallicity of LRGs extracted from the MS we built spectra of these galaxies using Bruzual \& Charlot (2003) (BC2003) model. When we fitted the spectra using the SSP library, we did not find that the measured time intervals correlated with the known time intervals. However, when using our model spectra (and the known mass-weighted-ages from the simulation) for age-dating, the systematic and statistical errors were significantly reduced (see Tab. 1). We are currently carrying out a much more thorough analysis of the uncertainties involved in age-dating.

Table 1. Errors from the Age Dating, comparing SSP ages with ages using our model spectra

\begin{tabular}{cccccccc}
\hline & \multicolumn{3}{c}{ SSP Fit } & \multicolumn{3}{c}{ Model Fit } \\
$\mathrm{z}$ & age & age & $\Delta^{b}$ & $\sigma^{c}$ & age & $\Delta^{b}$ & $\sigma^{c}$ \\
\hline 0.32 & 10.08 & 8.15 & 0.14 & 1.85 & 8.32 & 0.01 & 0.29 \\
0.46 & 8.95 & 7.42 & 0.20 & 1.72 & 7.22 & 0.01 & 0.33 \\
0.51 & 8.59 & 7.06 & 0.23 & 1.65 & 6.83 & 0.02 & 0.33 \\
0.56 & 8.25 & 6.62 & 0.16 & 1.69 & 6.48 & 0.01 & 0.33 \\
\hline
\end{tabular}

Notes:

a Age of the Universe in Gyrs.

${ }^{b}$ Mean difference between measured age and mass-weighted age.

$c$ Standard deviation of difference between measured age and mass-weighted age.

\section{Observing Program}

We estimated the error on individual ages of galaxies as a function of signal-to-noise and resolution using Monte Carlo simulations of spectral fitting. We then estimated the error on the mean age at a given redshift as a function of the number of galaxies observed and the error on ages of individual galaxies. We estimate that a $10 \%$ uncertainty on $\mathrm{H}(\mathrm{z})$ at $z \sim 0.42$ is obtainable with less than 20 hours of time on SALT while a $3 \%$ uncertainty requires almost 200 hours.

\section{References}

Bruzual, G. \& Charlot, S. 2003, MNRAS, 344, 1000

Crawford, S. M. et al. 2009, MNRAS submitted

Eisenstein, D. J. et al. 2001, AJ, 122, 2267

Jimenez, R. \& Loeb, E. 2002, ApJ, 593, 622 